\title{
Caracterización de enfermedades neurológicas en caninos: Universidad de San Carlos de Guatemala, año 2017
}

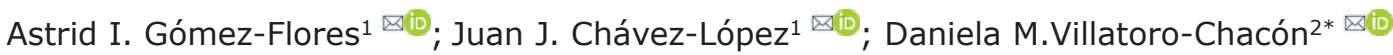

\begin{abstract}
${ }^{1}$ Universidad de San Carlos de Guatemala, Facultad de Medicina Veterinaria y Zootecnia, Hospital Veterinario, Departamento de Clínica de Animales de Compañía, ciudad de Guatemala, Guatemala.

2Universidad de San Carlos de Guatemala, Facultad de Medicina Veterinaria y Zootecnia, Hospital Veterinario, Departamento de Ayudas Diagnósticas, ciudad de Guatemala, Guatemala.

*Correspondencia: danavilla47@gmail.com
\end{abstract}

Recibido: Junio 2020; Aceptado: Abril 2021; Publicado: Agosto 2021.

\section{RESUMEN}

Objetivo. Determinar la casuística de enfermedades neurológicas, así como la edad, raza y los sitios neuroanatómicos afectados. Materiales y métodos. Se realizó un estudio retrospectivo de los expedientes clínicos de los pacientes caninos atendidos en el Hospital Veterinario de la Facultad de Medicina Veterinaria y Zootecnia de la Universidad de San Carlos de Guatemala en el año 2017. Los datos fueron clasificados según la regla nemotécnica VITAMIN D. Se incluyeron las variables sexo, raza, edad y localización neuroanatómica de lesiones. Los datos fueron resumidos utilizando estadística descriptiva por medio de tablas de distribución de frecuencias. Resultados. De los 1127 registros de casos la prevalencia de enfermedades neurológicas fue de $8.43 \%$. La frecuencia fue mayor en machos que en hembras (53.68\% vs $46.32 \%$ ). El grupo etario más afectado oscila entre los cero y los siete años. Respecto a razas, se observó una mayor frecuencia en caninos sin raza definida $(25.53 \%)$ seguido de los perros French Poodle $(20.21 \%)$. Las patologías encontradas fueron: vascular $(1.05 \%)$, inflamatoria/ infecciosa $(25.26 \%)$, traumática $(13.68 \%)$, metabólica $(8.42 \%)$, idiopática $(6.32 \%)$, neoplásica $(1.05 \%)$ y degenerativa $(44.21 \%)$. La neurolocalización más frecuente fue a nivel de SNC (86.32\%). Conclusiones. Según los hallazgos de este estudio se determinó que las enfermedades de carácter degenerativo representaron la mayor parte de la casuística neurológica. La mayor frecuencia de casos se presentó en pacientes caninos jóvenes, machos de razas mixtas. El sitio neuroanatómico más frecuente de localización de lesiones fue en medula espinal a nivel toracolumbar.

Palabras clave: Clasificación de enfermedades; neurología; Guatemala (Fuente: DeCS, MeSH).

\section{ABSTRACT}

Objetive. To determine the casuistry of neurological diseases, as well as the age, race and affected neuroanatomical sites. Materials and methods. A retrospective study of the clinical records of canine patients attended was carried out at the Veterinary Hospital of the Faculty of Veterinary Medicine and Zootechnics of the University Universidad de San Carlos de Guatemala in 2017. The data were classified according to the VitaminD mnemonic rule. The variables sex, race, age, and neuroanatomical

Como citar (Vancouver).

Gómez-Flores AI, Chávez-López Jj, Villatoro-Chacón DM. Caracterización de enfermedades neurológicas en caninos: Universidad de San Carlos de Guatemala, año 2017. Rev MVZ Córdoba. 2021; 26(3):e2047. https://doi.org/10.21897/rmvz.2047 CC) $\$$ CEl (los) autor (es) 2021. Este artículo se distribuye bajo los términos de la licencia internacional Creative Commons Attribution 4.0 (https:// BY NC SA siempre y cuando den crédito y licencien sus nuevas creaciones bajo las mismas condiciones. 
localization of lesions were included. The data were summarized using descriptive statistics through frequency distribution tables. Results. From 1127 case records, the prevalence of neurological diseases was $8.43 \%$. The frequency was higher in males than in females $(53.68 \%$ vs $46.32 \%)$. The most affected age group ranges from zero to seven years. Regarding breeds, a higher frequency was observed in canines without defined breed (25.53\%) followed by French Poodle dogs $(20.21 \%)$. The pathologies found were vascular $(1.05 \%)$, inflammatory/infectious $(25.26 \%)$, traumatic $(13.68 \%)$, metabolic $(8.42 \%)$, idiopathic $(6.32 \%)$, neoplastic $(1.05 \%)$ and degenerative $(44.21 \%)$. The most common neurolocalization was at the CNS level (86.32\%). Conclusions. According to the findings of this study, it was determined that degenerative diseases represented most of the neurological casuistry. The highest frequency of cases occurred in young canine patients, males of mixed breeds. The most frequent neuroanatomical site of lesions was in the spinal cord at the thoracolumbar level.

Keywords: Classification diseases; neurology; Guatemala (Fuente: DeCS, MeSH).

\section{INTRODUCCIÓN}

La evaluación neurológica es una herramienta que nos permite conocer la estructura y funcionamiento del sistema nervioso para realizar una correcta interpretación y localización específica de lesiones (1). Una vez establecido el sitio neuroanatómico de la lesión se debe categorizar la enfermedad, lo cual es importante para enlistar los diagnósticos diferenciales. Uno de los sistemas mayormente utilizados es la regla nemotécnica VITAMIN D (2) que se basa en los mecanismos fisiopatológicos de las enfermedades ya que trata de establecer un orden de las patologías tomando en cuenta la edad, raza, sexo, anamnesis y examen físico (3).

El clínico debe considerar a su vez que cada categoría de enfermedad involucra un rango etario y una predilección racial, entre otros factores a considerar. Por ejemplo, Mondino et al (4) indican que las patologías neurológicas que se presentan en los perros con mayor frecuencia son mielopatías, encefalopatías, encefalomielopatías, síndromes vestibulares y cerebelosos. Por otra parte, Fluehmann et al (5) y Pellegrino et al (2) reportan la epilepsia idiopática, hidrocefalia, neoplasias cerebrales y enfermedades del disco intervertebral. Dada la diversidad de la casuística de cada lugar, es necesario conocer la presentación de las enfermedades en cada contexto clínico.

El propósito del presente estudio fue generar información acerca de las principales patologías que afectan al sistema nervioso en los pacientes atendidos en el Hospital Veterinario de la Facultad de Medicina Veterinaria y Zootecnia de la Universidad de San Carlos de Guatemala. Esto con la finalidad de dar a conocer a los clínicos veterinarios las presentaciones más comunes de la enfermedad en el área de estudio.

\section{MATERIALES Y MÉTODOS}

Tipo de estudio. Se realizó un estudio retrospectivo a partir de registros de 1127 casos clínicos de pacientes caninos.

Localización. Los pacientes fueron atendidos en el Hospital Veterinario de la Facultad de Medicina Veterinaria y Zootecnia (FMVZ) de la Universidad de San Carlos de Guatemala (USAC) cuyas coordenadas son $14034^{\prime} 58.44^{\prime} \mathrm{N}$ y $90^{\circ} 33^{\prime} 10.44$ W durante el año de 2017.

Toma de datos. Se tomaron registros de las variables: sexo, raza, edad y localización neuroanatómica de lesiones de pacientes caninos con trastornos neurológicos atendidos en consulta neurológica. La clasificación de etiologías se realizó acorde a la regla nemotécnica VITAMIND mediante su acrónimo: V, vascular; I, inflamatorio, infeccioso; T, traumático, toxico; A, anómalo; $M$, metabólico; I, idiopático; N, neoplásico; y D, Degenerativo (3). Los pacientes fueron agrupados de acuerdo a su edad en cuatro rangos etarios: 0 a 3 años, 4 a 7 años, 8 a 11 años y 12 a 15 años de edad.

Los diagnósticos fueron emitidos utilizando imágenes radiológicas simples, ecografía, pruebas de laboratorio tales como hemograma completo, bioquímica sanguínea, urianálisis y pruebas de inmunoensayo cromatográfico. En los casos en los que no fue posible realizar ayudas diagnósticas se utilizó el diagnóstico presuntivo establecido a través de hallazgos de lesiones y signos en el examen físico y neurológico, así como el razonamiento clínico a través del inicio y progresión de la enfermedad.

Análisis de datos. Para el análisis de datos se utilizó estadística descriptiva, empleando 
tablas de distribución de frecuencias a través del programa estadístico past ${ }^{\circledR}$ versión 3 .

\section{RESULTADOS}

Se examinaron 1,127 registros de casos. La prevalencia de enfermedades neurológicas en la muestra estudiada fue de $8.43 \%$. En cuanto al sexo el $53.68 \%(n=51)$ correspondió a machos y el $46.32 \%(n=44)$ a hembras. La edad media de los pacientes con enfermedad neurológica fue de $5.60 \pm 0.42$ años. Sin embargo, dada la distribución de las frecuencias se categorizó la variable edad en cuatro grupos (Tabla 1) siendo los pacientes cachorros y adultos jóvenes los grupos con mayores frecuencias.

Tabla 1. Distribución de pacientes con enfermedad neurológica según grupo etario.

\begin{tabular}{ccc}
\hline Años & n & \% \\
\hline 0 a 3 & 34 & 35.79 \\
4 a 7 & 30 & 31.58 \\
8 a 11 & 21 & 22.11 \\
12 a 15 & 10 & 10.53 \\
\hline
\end{tabular}

En cuanto a la raza, la casuística de enfermedad neurológica se observó con mayor frecuencia en pacientes sin raza definida (25.53\%) seguido de los pacientes de raza French Poodle (20.21\%) (Tabla 2), otras razas constituyeron el $31.91 \%$.

Tabla 2. Frecuencia de casos de caninos que presentaron enfermedad neurológica, según su raza.

\begin{tabular}{ccc}
\hline Raza & $\mathbf{n}$ & $\mathbf{\%}$ \\
\hline Sin raza definida & 25 & 25.53 \\
French Poodle & 19 & 20.21 \\
Schnauzer & 7 & 7.45 \\
Husky Siberiano & 7 & 7.45 \\
Cocker Spaniel & 7 & 7.45 \\
Otras & 30 & 31.91 \\
\hline
\end{tabular}

Categorización de enfermedades según sistema VITAMIN D. En la tabla 3 se determinó que las enfermedades de carácter degenerativo representaron la mayor parte de la casuística neurológica (44.21\%), seguida de las de carácter inflamatorio/infeccioso (25.26\%) y traumático/ tóxico $(13.68 \%)$.
Tabla 3. Frecuencia de casos en caninos que presentaron enfermedad neurológica atendidos en el hospital veterinario de la FMVZ de la USAC, según categoría del sistema VITAMIND, durante 2017.

\begin{tabular}{ccc}
\hline VITAMIND & $\mathbf{n}$ & \% \\
\hline Vascular & 1 & 1.05 \\
Inflamatorio / Infeccioso & 24 & 25.26 \\
Traumático / tóxico & 13 & 13.68 \\
Alérgico / autoinmune / anomalía del desarrollo & 0 & 0 \\
Metabólico & 8 & 8.42 \\
Idiopático & 6 & 6.31 \\
Neoplásico / nutricional & 1 & 1.05 \\
Degenerativa & 42 & 44.21 \\
\hline
\end{tabular}

En la tabla 4 se presentan las frecuencias de los casos de las enfermedades neurológicas de cada categoría del sistema VITAMIN D.

Tabla 4. Frecuencia de casos de enfermedades neurológicas por cada categoría del sistema VITAMIN D.

\begin{tabular}{|c|c|c|}
\hline Enfermedades Neurológicas & $\mathbf{n}$ & $\%$ \\
\hline \multicolumn{3}{|l|}{ Vasculares } \\
\hline Accidente cerebrovascular & 1 & 100 \\
\hline \multicolumn{3}{|l|}{ Inflamatorias infecciosas } \\
\hline Distemper canino & 15 & 62.5 \\
\hline Ehrlichiosis & 2 & 8.3 \\
\hline Neosporosis & 1 & 4.17 \\
\hline Toxocariasis & 1 & 4.17 \\
\hline \multicolumn{3}{|l|}{ Inflamatorias no infecciosas } \\
\hline Encefalitis por causas desconocidas & 4 & 16.7 \\
\hline Poliomiositis & 1 & 4.16 \\
\hline \multicolumn{3}{|l|}{ Traumáticas o tóxicas } \\
\hline Trauma medular & 9 & 69.23 \\
\hline Trauma craneoencefálico & 2 & 15.38 \\
\hline Inestabilidad atlantoaxial & 1 & 7.69 \\
\hline Inestabilidad lumbosacra & 1 & 7.69 \\
\hline \multicolumn{3}{|l|}{ Anomalías } \\
\hline \multicolumn{3}{|l|}{ Metabólicas } \\
\hline $\begin{array}{l}\text { Encefalopatía hipertensiva por enfermedad } \\
\text { renal crónica }\end{array}$ & 6 & 75 \\
\hline Hipocalcemia & 1 & 12.5 \\
\hline Hipotiroidismo & 1 & 12.5 \\
\hline \multicolumn{3}{|l|}{ Idiopáticas } \\
\hline Epilepsia genética & 3 & 50 \\
\hline Parálisis facial & 1 & 16.66 \\
\hline Síndrome vestibular periférico & 2 & 33.34 \\
\hline \multicolumn{3}{|l|}{ Neoplásicas } \\
\hline Enfermedad neoplásica cerebral & 1 & 100 \\
\hline \multicolumn{3}{|l|}{ Degenerativas } \\
\hline Enfermedad del disco intervertebral & 22 & 52.38 \\
\hline Espondilosis deformante & 16 & 38.10 \\
\hline Calcificación de discos intervertebrales & 1 & 2.38 \\
\hline Mielopatía degenerativa & 1 & 2.38 \\
\hline Fusión de vertebras sacras & 1 & 2.38 \\
\hline Abiotrofia cerebelar & 1 & 2.38 \\
\hline
\end{tabular}


De la categoría degenerativa la principal enfermedad diagnosticada fue la enfermedad del disco intervertebral.

Localización neuroanatómica. En cuanto a la localización neuroanatómica, el $86.31 \%$ de los casos se presentó en el sistema nervioso central, mientras que el $2.11 \%$ en el sistema nervioso periférico. El $11.58 \%$ corresponden a trastornos sin localización neuroanatómica especifica.

A nivel del sistema nervioso central, el $42.68 \%$ de los casos estudiados fueron a nivel de la región intracraneal, mientras que el $57.32 \%$ a nivel de la región extracraneal. En la tabla 5 se presentan las frecuencias de los sitios neuroanatómicos de lesión intra y extracraneal.

Tabla 5. Frecuencia de casos en pacientes caninos con enfermedad neurológica a nivel de sistema nervioso central según sitio neuroanatómico.

\begin{tabular}{ccc}
\hline & n & \% \\
\hline Neurolocalización & \multicolumn{2}{c}{ Intracraneal } \\
\hline Cerebro & 25 & 30.4 \\
Cerebelo & 8 & 9.75 \\
Tallo & 0 & 0 \\
Vestibular central & 0 & 0 \\
Vestibular periférico & 2 & 2.44 \\
\hline Neurolocalización & \multicolumn{2}{c}{ Extracraneal } \\
& (médula espinal) \\
\hline Cervical & 9 & 10.98 \\
Cervicotoráxica & 0 & 0 \\
Toracolumbar & 28 & 34.15 \\
Lumbosacra & 10 & 12.19 \\
Cauda equina & 0 & 0 \\
\hline
\end{tabular}

Sólo el $2.11 \%$ de la casuística presentó lesión a nivel del sistema nervioso periférico siendo un caso reportado por parálisis facial y otro por polimiositis. En la tabla 6 se describen las frecuencias según el sitio neuroanatómico de la lesión.

Tabla 6. Frecuencia de casos en pacientes caninos con enfermedad neurológica a nivel de sistema nervioso periférico según sitio neuroanatómico.

\begin{tabular}{ccc}
\hline Neurolocalización & $\mathbf{n}$ & $\mathbf{\%}$ \\
\hline Raíces nerviosas & 0 & 0 \\
Nervios periféricos & 1 & 50 \\
Unión neuromuscular & 0 & 0 \\
Muscular & 1 & 50 \\
\hline
\end{tabular}

\section{DISCUSIÓN}

La prevalencia de enfermedades neurológicas encontrada brinda al médico clínico información epidemiológica para el diagnóstico según su entorno ya que son escasos los estudios que comparten este dato o bien estos son enfocados a una enfermedad en específico. Por otra parte, las enfermedades neurológicas con mayor casuística fueron las de carácter degenerativo por enfermedad del disco intervertebral. Pellegrino et al (2), Mondino et al (4), Fluehmann et al (5) y Chaves et al (6) reportan frecuencias similares. Esta enfermedad se presentó principalmente en caninos de razas de tamaño mediano y condrodistróficas de edades medias y avanzadas lo que concuerda con lo descrito por Fluehmann et al (5), Chaves et al (6) y Murakami et al (7). Afectando la región toracolumbar y cervicotorácica de la medula espinal, similar a lo reportado por Shimose y Salinas (8).

La segunda enfermedad de mayor prevalencia fue la espondilosis deformante. Los caninos más afectados fueron de edades avanzadas, lo cual coincide con lo observado por Pérez et al (9) y Kranenburg et al (10). Las razas más predisponentes a esta enfermedad son los pastores alemanes y los boxers (10) así como también las razas de trabajo o de deporte (11). Esto supone una diferencia con los resultados de este estudio, puesto que las razas más frecuentemente afectadas fueron caninos sin raza definida, French Poodle y Schnauzer. Esta diferencia puede deberse a que las razas mixtas o de tallas pequeñas-medianas son más comunes en Guatemala, pero igualmente están sujetos a ejercicios constantes o bien, pueden sufrir algún tipo de microtraumatismo que conlleve al desarrollo de la enfermedad. Los sitios neuroanatómicos afectados con mayor frecuencia fueron los segmentos medulares toracolumbar y lumbosacro, similar a lo reportado por Pérez et al (9) quienes demuestran que los sitios más frecuentes de localización de lesiones de espondilosis fueron a nivel lumbosacro, lumbar y torácico. Esta enfermedad afecta tanto a caninos machos como hembras.

La casuística de enfermedades de carácter infeccioso/inflamatorio más frecuente fue encefalitis por agentes infecciosos tales como distemper canino, erlichiosis, neosporosis y toxocariasis. Los caninos afectados fueron aquellos sin raza definida y French Poodle con rangos etarios entre 0 y 3 años y 4 a 7 años de 
edad. Sobre este aspecto Pellegrino et al (2) indica que la prevalencia de estos trastornos aumenta con la edad puesto que observó un $22 \%$ de casos en perros menores de 1 año, 33\% en animales de 1 a 6 años y $45 \%$ en edades superiores a 6 años. La alta casuística de enfermedades infecciosas especialmente el distemper canino en América Latina, al compararla con Europa o Norteamérica se debe a una mayor cantidad de perros abandonados que no reciben inmunización en forma sistemática (2).

Dentro de la categoría de enfermedades traumáticas del VITAMIND, el trauma medular fue la enfermedad diagnosticada de mayor prevalencia. Esto concuerda con Mendes y Arias (11) quienes observaron en su estudio que los segmentos medulares más afectados fueron el nivel toracolumbar y el lumbosacro con una mayor predominancia en caninos machos jóvenes, probablemente debido al comportamiento imprudente de estos animales. Las principales causas de trauma son atropellamientos, agresión por otro animal y caídas. Esto puede estar relacionado al elevado número de animales errantes, al alto flujo de vehículos y a la irresponsabilidad de propietarios que no emplean medidas de seguridad durante paseos, como el uso de correas (6).

El sitio neuroanatómico más frecuente de localización de lesiones fue en las estructuras extracraneales a nivel toracolumbar de la medula espinal coincidiendo con lo descrito por Arias et al (12) quienes mencionan que cerca del $85 \%$ de los canes con enfermedades a nivel de disco intervertebral presentan afecciones en la región toracolumbar y el $15 \%$ en la región cervical. Un factor para la presentación de lesiones a nivel toracolumbar puede ser la estrechez anatómica de su canal medular al compararlo con la región cervical (8).

El grupo etario más afectado fue el de 0 a 3 años. Las enfermedades neurológicas diagnosticadas en pacientes entre este rango de edad pertenecían a la categoría infecciosa/inflamatoria. Este grupo etario podría encontrarse en mayor riesgo de infección por agentes virales y protozoos probablemente relacionado a la inmadurez de su sistema inmunológico, sobre todo aquellos que no reciben algún tipo de inmunización. Por otra parte, el grupo etario correspondiente a caninos adultos de entre 4 y 7 años, corresponde principalmente a enfermedades de categoría degenerativa similar a lo reportado por Shimose y Salinas (8).
En cuanto el sexo de los pacientes, hubo una mayor frecuencia de casos en caninos machos respecto a las hembras diagnosticados principalmente por enfermedades de carácter degenerativo por enfermedad del disco intervertebral, similar a lo evaluado por Itoh et al (13) y Mondino et al (4). Hay un significativo factor de riesgo en los machos para las enfermedades neurológicas degenerativas, probablemente ligado a su mayor peso o a un supuesto efecto protector de los estrógenos frente a la degeneración del disco intervertebral (8). Itoh et al (13) por su parte, sugieren que las hormonas sexuales podrían influir en la causa de hernias del disco intervertebral a nivel toracolumbar.

Los pacientes sin raza definida presentaron una mayor frecuencia de enfermedades neurológicas. Pellegrino et al (2) describe que esta situación es notoria en los países latinoamericanos, donde muchos propietarios adoptan animales callejeros o provenientes de refugios en lugar de comprar perros de pedigrí, por libre elección o motivos económicos. La principal razón de la susceptibilidad de este grupo de animales puede deberse a deficiencias de salud y nutrición, además de que se encuentran expuestos a toda clase de peligros en el exterior. Por otra parte, la tenencia de razas como el French Poodle, Chihuahua y Schnauzer, puede atribuirse al espacio físico, cuidado y alimentación que estas razas requieren por ser de talla pequeñamediana versus tallas grandes (14).

Cabe mencionar es que en Guatemala se tiene la tendencia de atribuir a todos los pacientes con signos neurológicos, diagnósticos erróneos de categoría tóxica. Sin embargo, en este estudio no se observó ninguna enfermedad de esta categoría sino únicamente de carácter traumático. Por lo anterior, es importante realizar un buen examen clínico y neurológico del paciente, ya que las ayudas diagnósticas no son comúnmente realizadas en Guatemala debido a limitaciones económicas de los clientes o la falta de equipo en pequeñas clínicas veterinarias.

Con los resultados evidenciados en este estudio, el análisis de historia clínica, signología, neurolocalización, evolución y progresión de la enfermedad es suficiente para obtener un buen diagnóstico siempre y cuando se realice una práctica clínica fundamentada y razonada. El hecho de identificar la categoría de enfermedad es suficiente para iniciar un tratamiento adecuado y solucionar el problema neurológico, aun cuando no se pueda alcanzar el diagnóstico etiológico 
$(1,6)$. No obstante, se recomienda que siempre que se pueda tener acceso al uso de equipo de imágenes para obtener un diagnóstico exacto y con ello elegir el mejor tratamiento para la recuperación del paciente.

El presente estudio abre la puerta a futuras investigaciones de mayor duración y en otros lugares geográficos que amplíen la presentación epidemiológica de las enfermedades neurológicas. Así mismo es importante la constante capacitación y formación de los médicos en esta área para evitar errores diagnósticos y proporcionar un servicio de calidad a los pacientes utilizando los medios que se tengan en el entorno de trabajo.

\section{Conflicto de intereses}

Los autores declaramos que no existe conflicto de interés.

\section{REFERENCIAS}

1. Taylor S. Trastornos neuromusculares. En: Couto CG, Nelson RW. Medicina Interna de pequeños animales. 4ta ed. Barcelona España: Editorial Elsevier; 2010.

2. Pellegrino F, Pacheco E, Vazzoler $M$. Caracterización de los trastornos neurológicos en los perros: 1652 casos (marzo 2008-junio 2010). Parte 1. Rev Neurol Arg. 2011; 2(1):78-96. https://neurovetargentina.com. ar/revista/revista neurologia_02.pdf

3. Pellegrino F. Las claves del diagnóstico neurológico para el veterinario clínico. $1^{\mathrm{a}} \mathrm{ed}$. Buenos Aires Argentina: Inter Médica; 2014.

4. Mondino A, Piaggio J, Loureiro C, Vasconcellos $R$, Delucchi L. Efecto de la raza, sexo y edad en la presentación de enfermedades del sistema nervioso central en caninos atendidos en el Hospital de la Facultad de Veterinaria de Uruguay. Veterinaria (Montevideo). 2015; 51(199):4-11. http://www.revistasmvu.com. uy/index.php/smvu/article/view/119/64

5. Fluehmann G, Doherr M, Jaggy A. Canine Neurological diseases in a referral hospital population between 1989 and 2000 in Switzerland. J Small Anim Pract. 2006; 47(10):582-587. https://doi.org/10.1111/ j.1748-5827.2006.00106.x

6. Chaves RO, Beckmann DV, Santos RPD, Aiello $\mathrm{G}$, Andrades AO, Baumhardt R, et al. Doença neurológicas em cães atendidos no Hospital Veterinário da Universidade Federal de Santa Maria, RS: 1.184 casos (2006-2013). Pesq Vet Bras. 2014;34(10):996-1001. https://doi. org/10.1590/S0100-736X2014001000012

7. Murakami T, Feeney D, Willey J, Carlin B. Evaluation of the accuracy of neurologic data, survey radiographic results, or both for localization of the site of thoracolumbar intervertebral disk herniation in dogs. Am J Vet Med Res. 2014; 75(3):251-259. https:// doi.org/10.2460/ajvr.75.3.251
8. Shimose CP, Salinas CE. Caracterización de lesiones en columna vertebral mediante tomografía computarizada en canes de Lima, Perú. Rev Investig Vet Perú. 2018; 29(1):132141. http://dx.doi.org/10.15381/rivep. v29i1.14204

9. Pérez $M$, Verde $M T$, Unzueta $A$. Lesiones radiográficas y participación de factores individuales en la espondilosis deformante y la esclerosis vertebral en perros. Rev AVEPA. 2003; 23(1):18-24. https://ddd.uab.cat/pub/ clivetpeqani/11307064v23n1/11307064v23n 1p18.pdf

10. Kranenburg H, Voorhout G, Grinwis G, Hazewinkel $H$, Meji B. Diffuse idiopathic skeletal hyperostosis (DISH) and spondylosis deformans in purebred dogs: a retrospective radiographic study. Vet J. 2011; 190(2):e84-e90. https:// doi.org/10.1016/j.tvjl.2011.04.008

11. Mendes D, Arias M. Traumatismo da medula espinal em cães e gatos: estudo prospectivo de 57 casos. Pesq Vet Bras. 2012; 32(12):13041312. https://dx.doi.org/10.1590/S0100736X2012001200015

12. Arias M, Severo M, Tudury E. Trauma medular em cães e gatos: revisão da fisiopatologia e do tratamento médico. Semina. 2007; 28(1):115. https://doi.org/10.5433/1679$\underline{0359.2007 v 28 n 1 p 115}$

13. Itoh $\mathrm{H}$, Hara $\mathrm{Y}$, Yoshimi $\mathrm{N}$, Harada $\mathrm{Y}$, Nezu $Y$, Yogo $T$, et al. A Retrospective Study of Intervertebral Disc Herniation in Dogs in Japan: 297 Cases. J Vet Med Sci. 2008; 70(7):701706. https://doi.org/10.1292/jvms.70.701

14. Alvarado-Peréz A, Villatoro-Chacón D, ChávezLópez J, Arizandieta-Altán C. Caracterización de la población canina atendida en el centro municipal de atención canina de la ciudad de Guatemala. REDVET. 2017; 18(12):1-9. http://www.veterinaria.org/revistas/redvet 